\title{
EFICIENCIA PRODUCTIA EN SECTOR PORCINO: UN ESTUDIO DE CASOS DE DINAMARCA Y POLONIA
}

\author{
Juan Cabas-Monje ${ }^{a^{*}}$, Luis Améstica-Rivas ${ }^{a}$, Jonathan Labra-Hernández ${ }^{\text {, }}$ Bouali Guesmi ${ }^{\text {** }}$, José \\ María Gil ${ }^{b}$
}

${ }^{a}$ Facultad de Ciencias Empresariales, Departamento de Gestión Empresarial, Grupo de Investigación en Agronegocios. Universidad del Bío-Bío, Región de Ñuble, Chile. jcabas@ubiobio.cl; lamestica@ubiobio.cl; jlabra@ubiobio.cl. ${ }^{b}$ Center for Agro-Food Economics and Development (CREDAUPC-IRTA), Castelldefels, España.bouali.guesmi@upc.edu; chema.gil@upc.edu.

\section{RESUMEN}

Dentro del mercado europeo de carne de cerdo, Dinamarca y Polonia son dos actores relevantes que presentan algunas diferencias en sus sistemas productivos (SP). El presente trabajo estudia las variables que explican el comportamiento de la producción de carne porcina y la eficiencia técnica relativa de los SP tomando en cuenta las diferencias en los sistemas. Para ello, se utilizó modelos de frontera estocástica de producción del tipo trans-log, para muestras de productores porcinos en el periodo 2010-2015. Los resultados indican que las muestras de productores de ambos países presentan niveles de eficiencia altos, con valores de 0,899 y 0,859 para Dinamarca y Polonia, respectivamente. Los factores más importantes que explican la producción de cerdos son los alimentos y el capital. Además, Polonia presenta crecimiento tecnológico significativo en el periodo estudiado sin mostrar cambios en la eficiencia, mientras que, Dinamarca no presenta progreso tecnológico y adicionalmente muestra una baja en la eficiencia técnica relativa en el periodo.

Palabras clave: Eficiencia técnica; frontera estocástica; producción porcina

\section{INTRODUCCIÓN}

Polonia es el cuarto mayor productor europeo con un $8 \%$ de la producción del año 2019, mientras que, Dinamarca está en el sexto lugar con un 6\% [23]. Dinamarca y Polonia junto con la importancia que tienen en el mercado de carne porcina presentan ciertas características particulares en su SP y comercializador que resulta de interés estudiarlo. En Dinamarca, la industria porcina desempeña un papel relevante en la economía local, donde más del $90 \%$ de su producción se exporta a 120 mercados de todo el mundo, esto representa más del 19\% del total de productos alimentarios exportados [11]. Lideran en rendimiento por lechones destetados por cada cerda en reproducción al año (a), tienen un SP con el más avanzado desarrollo tecnológico y bajo estricta legislación ambiental y sanitaria, cada vez más concentrado en grandes explotaciones. Tienen una estructura cooperativa y integrada, en la que los criadores son propietarios de la explotación, la producción, los mataderos y las empresas de elaboración de alimentos. Polonia ha experimentado cambios estructurales importantes en las últimas dos décadas, aumentando la concentración de la producción en granjas de mayor tamaño. Pero, en comparación con otros países de la Unión Europea, la producción sigue presentando una estructura muy fragmentada con escalas de producción muy bajas, lo que lleva a importar grandes cantidades de lechones para la cría y carne principalmente desde Dinamarca. A lo anterior, se suma el hecho de una integración vertical y horizontal poco desarrollada, con escasa colaboración entre productores de cerdos y procesadores de carne. A pesar que, Polonia incrementó su participación en el mercado europeo pasando del 8\% en 2010 al 8\% en 2019 y Dinamarca la disminuyó desde un $7,5 \%$ a un $6 \%$ en 2019, se considera a Dinamarca como un referente mundial en términos de su SP y en su estructura cooperativa y a Polonia como un país que debe mejorar para alcanzar niveles de desarrollo superiores.

Como una forma de profundizar en el conocimiento de los SP de cerdos de ambos países, esta investigación tuvo como propósito estudiar las variables que explican el comportamiento de la producción de carne porcina y la eficiencia técnica relativa de los SP, que contribuya especialmente como otros estudios a la discusión sobre la eficiencia técnica en la producción porcina, considerando que la literatura en este tema es escasa y que en las estimaciones se han utilizado, tanto aproximaciones paramétricas de frontera estocástica [16, 26, 27], como no paramétricas (DEA) [7] y Análisis de Eficiencia Multidireccional [21].

\section{MATERIALES Y MÉTODOS}

La frontera estocástica de producción está definida en la ecuación (1): $Y_{i}=f\left(X_{i} ; \beta\right) e^{v-u}$, donde $v \sim N\left(0, \sigma_{v}^{2}\right)$, $\mathrm{u} \sim \mathrm{N}^{+}\left(0, \sigma_{\mathrm{u}}^{2}\right), \mathrm{i}=1,2, \ldots, \mathrm{n}$. $\mathrm{Y}_{\mathrm{i}}$ es la producción de carne de cerdo de cada individuo $\mathrm{i}, \mathrm{X}_{\mathrm{i}}$ es el vector de insumos del productor i, $\beta$ son los parámetros de factores estimados, $-\mathrm{u} \leq 0$ explica la ineficiencia técnica y v explica el ruido estadístico, ambos independientes entre sí. De tal forma, en esta investigación se utilizó una aproximación paramétrica para datos de panel desarrollados por Battese y Coelli [3, 4] que se muestra en la ecuación siguiente. 


$$
\begin{aligned}
& \operatorname{lny}_{\mathrm{kt}}=\beta_{0}+\beta_{1} \ln \ln \left(\text { feed }_{\mathrm{kt}}\right)+\beta_{2} \ln \ln \left(\mathrm{oi}_{\mathrm{kt}}\right)+\beta_{3} \ln \ln \left(\text { labor }_{\mathrm{kt}}\right)+\beta_{4} \ln \ln \left(\mathrm{cap} 1_{\mathrm{kt}}\right)+\beta_{5} \\
& \ln \ln \left(\operatorname{cap} 2_{\mathrm{kt}}\right)+\beta_{6} \text { mYear }+\beta_{11} \frac{\left(\ln \left(\mathrm{feed}_{\mathrm{kt}}\right)\right)^{2}}{2}+\beta_{22} \frac{\left(\ln \left(\mathrm{oi}_{\mathrm{kt}}\right)\right)^{2}}{2} \\
& +\beta_{33} \frac{\left(\ln \left(\text { labor }_{\mathrm{kt}}\right)\right)^{2}}{2}+\beta_{44} \frac{\left(\ln \left(\operatorname{cap} 1_{\mathrm{kt}}\right)\right)^{2}}{2}+\beta_{55} \frac{\left(\ln \left(\operatorname{cap} 2_{\mathrm{kt}}\right)\right)^{2}}{2}+\beta_{12} \ln \ln \left(\mathrm{feed}_{\mathrm{kt}}\right) \\
& \ln \ln \left(\mathrm{oi}_{\mathrm{kt}}\right)+\beta_{13} \ln \ln \left(\text { feed }_{\mathrm{kt}}\right) \ln \ln \left(\text { labor }_{\mathrm{kt}}\right)+\beta_{14} \ln \ln \left(\text { feed }_{\mathrm{kt}}\right) \ln \ln \left(\mathrm{cap}_{\mathrm{kt}}\right) \\
& +\beta_{15} \ln \ln \left(\mathrm{feed}_{\mathrm{kt}}\right) \ln \ln \left(\mathrm{cap} 2_{\mathrm{kt}}\right)+\beta_{23} \ln \ln \left(\mathrm{oi}_{\mathrm{kt}}\right) \ln \ln \left(\mathrm{labor}_{\mathrm{kt}}\right)+\beta_{24} \\
& \ln \ln \left(\mathrm{oi}_{\mathrm{kt}}\right) \ln \ln \left(\mathrm{cap}_{\mathrm{kt}}\right)+\beta_{25} \ln \ln \left(\mathrm{oi}_{\mathrm{kt}}\right) \ln \ln \left(\mathrm{cap} 2_{\mathrm{kt}}\right)+\beta_{34} \ln \ln \left(\text { labor }_{\mathrm{kt}}\right) \\
& \ln \ln \left(\operatorname{cap} 1_{\mathrm{kt}}\right)+\beta_{35} \ln \ln \left(\text { labor }_{\mathrm{kt}}\right) \ln \ln \left(\mathrm{cap} 2_{\mathrm{kt}}\right)+\beta_{45} \ln \ln \left(\mathrm{cap} 1_{\mathrm{kt}}\right) \\
& \ln \ln \left(\operatorname{cap} 2_{\mathrm{kt}}\right)+\theta \mathrm{t}+\mathrm{v}_{\mathrm{kt}}-\mathrm{u}_{\mathrm{kt}}
\end{aligned}
$$

Los datos de producción porcina son obtenidos desde la Farm Accountancy Data Network (FADN) para el periodo 2010 al 2015, constituyendo un panel no balanceado con 91 empresas de Polonia y 92 empresas de Dinamarca, con un total de 396 y 358 observaciones, respectivamente. La producción de cerdos medida como el ingreso total en euros. Se definen cinco factores productivos o insumos, el capital1, el capital2, el trabajo, alimento y otros insumos. El capital 1 comprende edificios, equipamiento, máquinas, tractores, etc. El capital2 incluye el número cerdos presentes en el sistema que son convertidos en unidades de livestock y expresada en euros. El trabajo se describe como el trabajo total expresado en AWU. El alimento de cerdos esta expresado en euros. Finalmente, la variable otros insumos comprende otros costos específicos del ganado (veterinario, compra ocasional de animales, marketing, gastos generales en euros). Todos los valores monetarios están expresados en euros del 2015.

\section{RESULTADOS Y DISCUSIÓN}

La Tabla 1 presenta los coeficientes estimados del modelo de frontera estocástica de producción para los dos países. Se observa que las estimaciones de las elasticidades de producción en las medias de los términos lineales son positivos y en un valor significativas al nivel del $1 \%$ o del $5 \%$. El efecto más importante corresponde al coeficiente del alimento (lfeed), con un valor de 0,363. El segundo en importancia es el capital2 (lcap2) con un valor de 0,289, les siguen en importancia el capital1, otros insumos y el trabajo con valores de $0,167,0,155$ y 0,081 , respectivamente. Es interesante observar, a pesar de ser significativo, la menor importancia del factor productivo trabajo en la producción porcina. La tasa anual de progreso tecnológico es significativa al nivel del $1 \%$, indicando un crecimiento tecnológico anual de $2,1 \%$ en la muestra de productores de Polonia. La sumatoria de los coeficientes lineales es levemente superior a la unidad, lo que refleja rendimientos ligeramente crecientes de escala en sus medias muestrales para estos productores.

La Tabla 2 presenta los coeficientes estimados con forma funcional Trans-log para Dinamarca. Los signos de los coeficientes lineales estimados son como se esperaba, es decir, positivos y significativos al nivel del $1 \%$, excepto para el coeficiente del cambio tecnológico que es no significativo. Al igual que en el modelo de Polonia, los efectos más importantes corresponden al coeficiente del alimento (lfeed) y al del capital2 (lcap2), con valores de 0,462 y 0,264, respectivamente. En cambio, el efecto del capital2 es muy parecido, les siguen en importancia el trabajo, otros insumos y el capital1, con elasticidades de producción en las medias de $0,145,0,119$ y 0,037 , respectivamente. Se puede observar que, en la muestra de productores de Dinamarca el factor productivo trabajo tiene mayor efecto que en el modelo polaco. La sumatoria de los coeficientes lineales y el rechazo de la hipótesis nula de rendimientos constantes de escala, evidencian que las muestras de productores de Dinamarca presentan rendimientos constantes de escala en sus medias muestrales. La tasa anual de cambio tecnológico de alrededor del $0,6 \%$ no es significativa lo que expresa que la tecnología de producción no cambia en el tiempo. El coeficiente estimado de la variable time es negativo y significativo al 1\%, lo que significa que la eficiencia está decreciendo en el tiempo (Tabla 1).

La eficiencia técnica promedio para los productores de Polonia de esta muestra de productores porcinos llega a 0,859 , un valor que se podría denominar medio alto, con el $75 \%$ de los productores con eficiencia sobre 0,801 . La eficiencia técnica promedio de esta muestra de productores porcinos de Dinamarca llega a 0,899 , lo que es un valor alto y superior al encontrado para los productores de Polonia.

Tabla 1. Estimación frontera de posibilidades de producción polonia y dinamarca

\begin{tabular}{lcc}
\hline & Trans-log- Polonia & Translog - Dinamarca \\
\hline (Intercept) & $0,074(0,022)^{* * *}$ & $0,103(0,013)^{* * *}$ \\
\hline Lfeed & $0,363(0,029)^{* * *}$ & $0,462(0,023)^{* * *}$ \\
\hline Loi & $0,155(0,029)^{* * *}$ & $0,119(0,033)^{* * *}$ \\
\hline Llabor & $0,081(0,034)^{* *}$ & $0,145(0,027)^{* * *}$ \\
\hline lcap1 & $0,167(0,029)^{* * *}$ & $0,037(0,013)^{* * *}$ \\
\hline lcap2 & $0,289(0,033)^{* * *}$ & $0,264(0,042)^{* * *}$ \\
\hline
\end{tabular}




\begin{tabular}{|c|c|c|c|c|}
\hline \multirow{2}{*}{$\begin{array}{l}\text { mYear } \\
\mathrm{I}\left(0.5 *(\text { lfeed })^{\wedge} 2\right)\end{array}$} & \multicolumn{2}{|r|}{$0,021(0,005) * * *$} & $0,006(0,005)$ & \\
\hline & \multicolumn{2}{|r|}{$0,436(0,081)^{* * *}$} & $0,454(0,085)^{* * *}$ & \\
\hline \multicolumn{2}{|l|}{$\mathrm{I}\left(0.5 *(1 \mathrm{loi})^{\wedge} 2\right)$} & $0,070(0,067)$ & $-0,152(0,18)$ & \\
\hline \multicolumn{2}{|l|}{$\mathrm{I}\left(0.5 *(\text { llabor })^{\wedge} 2\right)$} & $0,061(0,116)$ & $0,044(0,104)$ & \\
\hline \multicolumn{2}{|l|}{$\mathrm{I}(0.5 *(\operatorname{lcap} 1 \wedge 2))$} & $0,085(0,067)$ & $0,014(0,006) * *$ & \\
\hline \multicolumn{2}{|l|}{$\mathrm{I}\left(0.5 *(\operatorname{lcap} 2)^{\wedge} 2\right)$} & $0,236(0,055) * * *$ & $0,062(0,106)$ & \\
\hline \multicolumn{2}{|l|}{ I(lfeed * loi) } & $-0,072(0,054)$ & $-0,151(0,083)^{*}$ & \\
\hline \multicolumn{2}{|l|}{$\mathrm{I}($ loi $*$ llabor $)$} & $-0,021(0,059)$ & $-0,011(0,096)$ & \\
\hline \multicolumn{2}{|l|}{ I(lfeed * llabor $)$} & $-0,114(0,065)^{*}$ & $-0,09(0,068)$ & \\
\hline \multicolumn{2}{|l|}{ I(lcap2 * lfeed $)$} & $-0,296(0,058) * * *$ & $-0,338(0,083) * * *$ & \\
\hline \multicolumn{2}{|l|}{ I(lcap2 * llabor) } & $0,093(0,065)$ & $0,109(0,11)$ & \\
\hline I(lcap2 * loi) & & $0,037(0,049)$ & $0,299(0$, & \\
\hline I(lcap1 * lcap2) & & $0,068(0,049)$ & $-0,054(0$ & \\
\hline I(lcap1 * lfeed) & & $-0,013(0,051)$ & $-0,003(0$ & \\
\hline I(lcap1 * loi) & & $-0,078(0,057)$ & $0,02(0$ & \\
\hline I(lcap1 * llabor) & & $0,003(0,059)$ & $-0,006(0$ & \\
\hline sigmaSq & & $0,054(0,008) * * *$ & $0,034(0,0)$ & \\
\hline Gamma & & $0,715(0,052)^{* * *}$ & $0,876(0,02$ & \\
\hline Time & & - & $-0,13(0,03$ & \\
\hline Lambda & & $1,585(0,200)^{* * *}$ & $2,662(0,3$ & \\
\hline $\begin{array}{l}\text { Los errores estándar } \\
\text { respectivamente. }\end{array}$ & tran entre & paréntesis. $*, * *$ y $* * *$ & significancia & 0,05 y 0,01 \\
\hline $\begin{array}{l}\text { La distribución de } 1 \\
\text { rangos superiores y } \\
\text { media en el periodo } \\
\text { explicada por la an } \\
\text { del valor mínimo } \\
\text { significancia del co }\end{array}$ & $\begin{array}{l}\text { la prias pres } \\
\text { lio, desde } \\
\text { del range } \\
863 \text { hast } \\
\text { de la vari }\end{array}$ & $\begin{array}{l}\text { enta una gran concentr } \\
\text { on inferior del rango. A } \\
0,937 \text { el año } 2010 \text { has } \\
\text { de valores de la efici } \\
\text { a } 0,667 \text {. Además, lo } \\
\text { lación de la eficiencia }\end{array}$ & $\begin{array}{l}\text { los producto } \\
\text { es clara la dis } \\
\text { el año } 2015 \\
\text { pecialmente, } \\
\text { do es consis } \\
\text { npo. }\end{array}$ & $\begin{array}{l}\text { encias en los } \\
\text { la eficiencia } \\
\text { on puede ser } \\
\text { disminución } \\
\text { signo y la }\end{array}$ \\
\hline TABLA 2. Resume & cias prod & uctores dinamarca por & & \\
\hline \begin{tabular}{|c|} 
Año \\
\end{tabular} & Mínimo & \begin{tabular}{l|r|} 
& Mediana \\
\end{tabular} & Media & Máximo \\
\hline 2010 & 0,8634 & 0,9366 & 0,9323 & 0,9897 \\
\hline 2011 & 0,7712 & 0,9276 & 0,9183 & 0,9934 \\
\hline 2012 & 0,7439 & 0,9195 & 0,9102 & 0,9925 \\
\hline 2013 & 0,7140 & 0,9043 & 0,8958 & 0,9848 \\
\hline 2014 & 0,6814 & 0,8955 & 0,8839 & 0,9818 \\
\hline 2015 & 0,6677 & 0,8992 & 0,8759 & 0,9889 \\
\hline
\end{tabular}

\section{CONCLUSIONES}

Los resultados de este estudio muestran que, a pesar de las diferencias estructurales que presentan ambos países, la eficiencia técnica relativa media de los productores porcinos en Polonia y Dinamarca es media alta, concretamente de 0,859 y 0,899 , respectivamente. A pesar de esto, los niveles de eficiencia podrían mejorarse si los productores realizan combinaciones óptimas de los factores productivos que beneficien la producción porcina en el territorio. Es interesante el hecho que los productores de Polonia presentan un progreso tecnológico anual constante de $2,1 \%$, sin cambio de eficiencia en el periodo. Esto puede ser el efecto de cambios estructurales en el SP, que aumentaron la productividad, pero no tuvieron efectos significativos en la eficiencia. Mientras tanto, la muestra de productores de Dinamarca no muestra cambios significativos en el progreso tecnológico en el tiempo, pero si una disminución significativa de la eficiencia. Esta situación, junto con factores relacionados al mercado, podría explicar la baja en la producción de carne de cerdo en el periodo de estudio. Finalmente, este estudio es importante para expandir la literatura en la eficiencia técnica de la producción porcina. Sin embargo, quedan para posteriores investigaciones determinar los factores que afectan o explican el cambio en la media de la eficiencia técnica, por ejemplo, las causas que subyacen a la caída en la eficiencia en la producción porcina en Dinamarca, y la incorporación del riesgo de producción en el análisis. Esto permitiría enfocar los aspectos de perfeccionamiento en la labor de los productores porcinos, y a su vez, generar y aplicar políticas públicas de apoyo a los productores que contribuyan a mejorar su competitividad y recuperar los niveles de producción y eficiencia de inicio de la década.

\section{REFERENCIAS BIBLIOGRAFICAS}


Battese, G.E. y Coelli, T.J. (1992). "Frontier production functions, technical efficiency and panel data: With application to paddy farmers in India". Journal of Productivity Analysis, 3:153-169.

Battese, G. E. y Coelli, T. J. (1995). “A model for technical inefficiency effects in a stochastic frontier production function for panel data". Empirical Economics. 20: 325-32.

Calafat, C., Martí, M. y Puertas, R. (2018). Eficiencia del sector ganadero valenciano: Aproximación no paramétrica. Información Técnica Económica Agraria, 114(1): 61-77.

DAFC (2018). Fakta Om Erhvervet 2017: Fødevareklyngen Har Kurs Mod Fremtiden (Facts and figures 2017, the food cluster is heading for the future). Copenhague, $40 \mathrm{pp}$.

Food and Agriculture Organization of the United Nation (FAO). Food Outlook - Biannual Report on Global Food Markets. (2020). Food Outlook, 1. Rome. https://doi.org/10.4060/ca9509en.

Labajova, K., Hansson, H., Asmild, M., Göransson, L., Lagerkvist, C. J. y Neil, M. (2016). "Multidirectional analysis of technical efficiency for pig production systems: The case of Sweden". Livestock Science, 187: 168-180.

Ministerio de Agricultura, Pesca y Alimentación, Gobierno de España. El sector de la carne de cerdo en cifras: Principales Indicadores Económicos. (2020). Subdirección General de Producciones Ganaderas y Cinegéticas, Dirección General de Producciones y Mercados Agrarios. 63 pp. 